\title{
SPECT/CT preoperatorio, una nueva herramienta diagnóstica para localizar adenomas paratiroideos. Presentación de 2 casos clínicos*
}

\author{
Drs. ROSSANA PRUZZO C. ${ }^{1}$, HORACIO AMARAL P. ${ }^{1}$, RICARDO ROSSI F. ${ }^{2}$, \\ BÁRBARA MORALES K. ${ }^{1}$, SRTA.M. EUGENIA MARTÍNEZ G. ${ }^{1}$ \\ 1 Servicio de Medicina Nuclear Departamento de Imágenes. \\ 2 Departamento de Cirugía. \\ Clínica Alemana de Santiago, Facultad de Medicina Universidad del Desarrollo. \\ Santiago, Chile.
}

\begin{abstract}
Localization of functioning parathyroid adenomas by SPECT/CT. Report of two cases

Single photon emission computed tomography and computed tomography (SPECT/CT), integrates a gamma camera and a CT scan and is effective for the location of parathyroid adenomas. We report a 55 years old male and a 80 years old female with primary hyperparathyroidism. In both cases the ${ }^{99 m} \mathrm{Tc}$-Sestamibi parathyroid scintigraphy detected a functioning nodule whose presence was confirmed with SPECT/CT.
\end{abstract}

Key words: Hyperparathyroidism, SPECT/CT, parathyroid adenoma.

\section{Resumen}

El SPECT/CT integra una gamacámara y un escáner radiológico en un solo equipo híbrido que fusiona la imagen de la cintigrafía SPECT (tomografía computada de fotón único) con la imagen morfológica obtenida con un escáner de baja intensidad, sin movilizar al paciente, en una perfecta correspondencia anátomofuncional, permitiendo identificar con exactitud la localización de un adenoma paratiroideo. Se describen dos pacientes con hiperparatiroidismo primario, con ecotomografía convencional negativa, donde el SPECT/CT demostró la localización exacta de adenomas de ubicación no habitual.

Palabras clave: SPECT, SPECT/CT, adenomas, paratiroides.

\section{Introducción}

Las modalidades diagnósticas para detectar tejido paratiroideo hiperfuncionante han estado evolucionado constantemente, utilizándose hoy en día principalmente la cintigrafía con ${ }^{99 \mathrm{~m}} \mathrm{Tc}$-Sestamibi en dos fases asociada a una imagen morfológica, habitualmente una ecotomografía ${ }^{1}$.
La cintigrafía con ${ }^{99 \mathrm{~m}} \mathrm{Tc}$-Sestamibi permite identificar tejido paratiroideo hiperfuncionante debido a una elevada captación del radiotrazador producto de una combinación de mayor flujo sanguíneo, tamaño $\mathrm{y}$ actividad mitocondrial ${ }^{2}$. Esta tiene una sensibilidad promedio de $87 \%$ para adenomas paratiroideos, como fue demostrado en un meta-análisis realizado en 894 pacientes ${ }^{3}$.

*Recibido el 26 de Octubre de 2009 y aceptado para publicación el 29 de Noviembre de 2009.

Correspondencia: Dra. Rossana Pruzzo C.

Vitacura 5951, Santiago, Chile.

E-mail: rpruzzo@alemana.cl 
La cintigrafía con ${ }^{99 m}$ Tc-Sestamibi mejora sustancialmente la localización en estudios pre-operatorios y ha aumentado la tasa de éxito de la cirugía mínimamente invasiva $a^{4}$ y selectiva ${ }^{5}$. Sin embargo, la falta de referencias anatómicas de la cintigrafía es una limitante, lo que ha llevado al desarrollo de un nuevo equipo conocido como SPECT/CT. Éste ha sido recientemente incorporado en nuestro país, e integra una gamacámara y un escáner radiológico en un solo equipo híbrido que fusiona la imagen de la cintigrafía SPECT (tomografía computada de fotón único) con la imagen morfológica obtenida con un escáner de baja intensidad, sin movilizar al paciente, en una perfecta correspondencia anátomo-funcional, permitiendo identificar con exactitud la localización de un adenoma paratiroideo ${ }^{6,7}$.

A continuación se describen dos pacientes con hiperparatiroidismo primario, con ecotomografía convencional negativa, donde el SPECT/CT demostró la localización exacta de adenomas de ubicación no habitual.

\section{Caso clínico 1}

Paciente varón de 55 años, presenta un cuadro clínico de litiasis renal derecha confirmada por

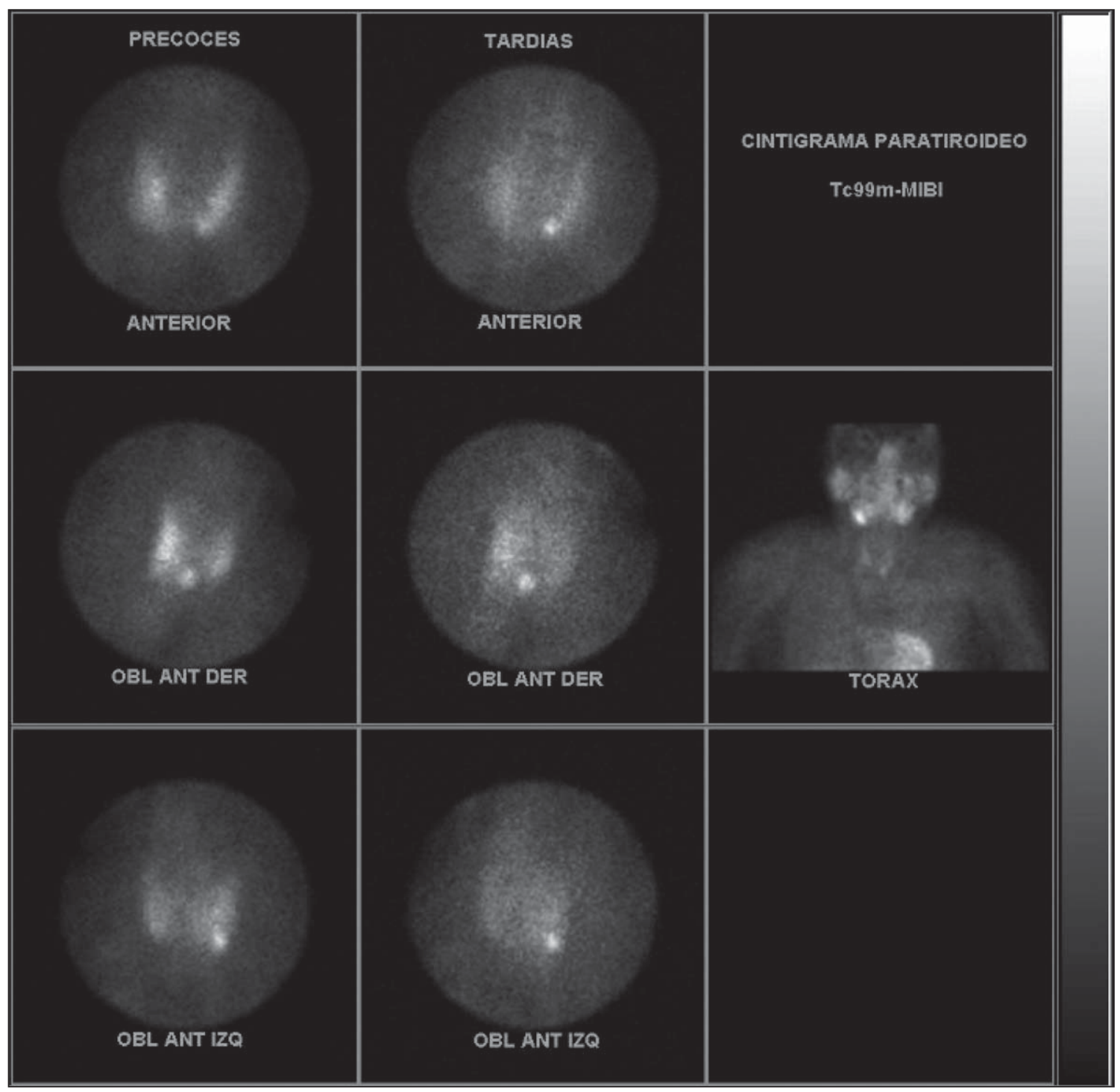

Figura 1. Caso 1: Cintigrama de paratiroides efectuado con colimador de pinhole y planar de tórax. Posterior al polo inferior del lóbulo tiroideo izquierdo se aprecia un foco hipercaptante, que es más evidente en el control tardío. 
pieloTAC. Una calcemia mostró un valor de 11,4 ng/ dL, una fosfemia de 3,4 y una PTH intacta de 124,4 $\mathrm{pg} / \mathrm{mL}$, con función renal normal, diagnosticándose un hiperparatiroidismo primario. Se efectuó una cintigrafía de paratiroides post-inyección e.v. de $20 \mathrm{mCi}$

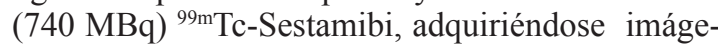
nes precoces y tardías de 15 y 90 minutos en una gamacámara con colimador de "pinhole", en proyecciones anterior y oblicuas anteriores, observándose un nódulo hipercaptante en situación posterior y caudal al lóbulo tiroideo izquierdo compatible con tejido paratiroideo hiperfuncionante (Figura 1). Se complementó con SPECT/CT sin medio de contraste

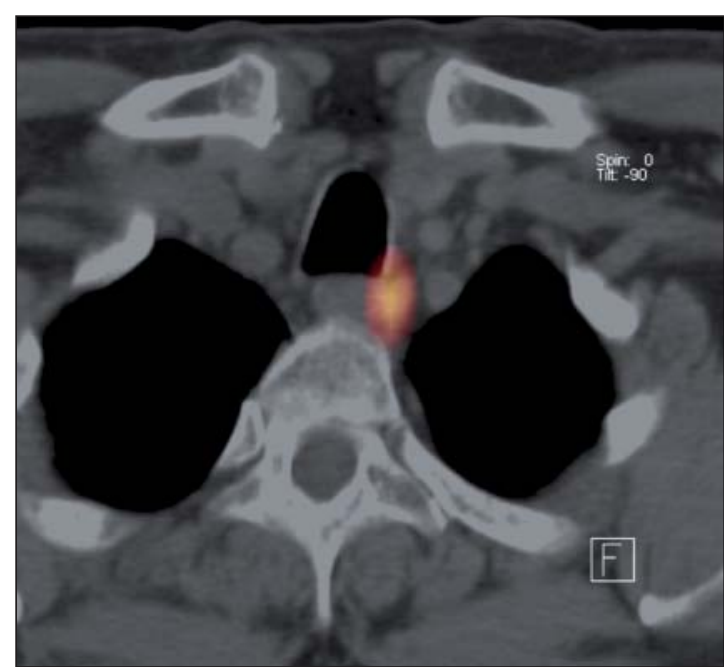

Figura 2. Caso 1: SPECT/CT. Imagen de fusión de la tomografía axial computada (CT) con los cortes tomográficos de la cintigrafía de paratiroides con Tc99m Sestamibi (SPECT). El foco hipercaptante corresponde a la localización del adenoma paratiroideo. yodado, identificándose un nódulo hipercaptante de ${ }^{99 m} \mathrm{Tc}-S e s t a m i b i$, de $15 \mathrm{~mm}$, situado en región postero-lateral a la tráquea, paraesofágico izquierdo (Figura 2). La ecotomografía convencional con transductor lineal 12 fue de características normales. En conocimiento de los hallazgos de la cintigrafía, se realizó un "second look" utilizándose un transductor sectorial lográndose identificar un nódulo hipoecogénico con vaso periférico al Doppler color.

Se realiza una exploración quirúrgica izquierda, extirpándose en región caudal un nódulo de $15 \mathrm{~mm}$ que se envía a biopsia diferida. Veinte minutos más tarde se obtuvo una PTH intacta, la que dio un valor de $30,9 \mathrm{pg} / \mathrm{mL}$. Calcemia post-operatoria $9,1 \mathrm{mg} / \mathrm{dL}$.

El estudio anatomo-patológico demostró una lesión nodular delimitada por una cápsula fina fibrosa de $26 \times 14 \times 5 \mathrm{~mm}$, de 0,855 gramos, compatible con adenoma paratiroideo.

\section{Caso clínico 2}

Mujer de 80 años, asintomática, presenta una hipercalcemia, hipofosfemia y PTH elevada, diagnosticándose un hiperparatiroidismo primario. Una ecotomografía demostró un adenoma paratiroideo de $8 \mathrm{~mm}$, caudal derecho, resecándose quirúrgicamente demostrándose histológicamente un adenoma paratiroideo.

Por persistencia de PTH y calcemia elevadas se realiza una cintigrafía de paratiroides con ${ }^{99 \mathrm{~m}} \mathrm{Tc}$ Sestamibi identificándose un foco hipercaptante posterior y caudal al lóbulo tiroideo derecho (Figura 3). Un SPECT/CT confirmó un nódulo hipercaptante de $26 \mathrm{~mm}$ situado en mediastino posterior (Figura 4), resecándose quirúrgicamente y descartándose lesiones paratiroideas contralaterales. Se utilizó una sonda "gamma-probe", demostrándose un mayor
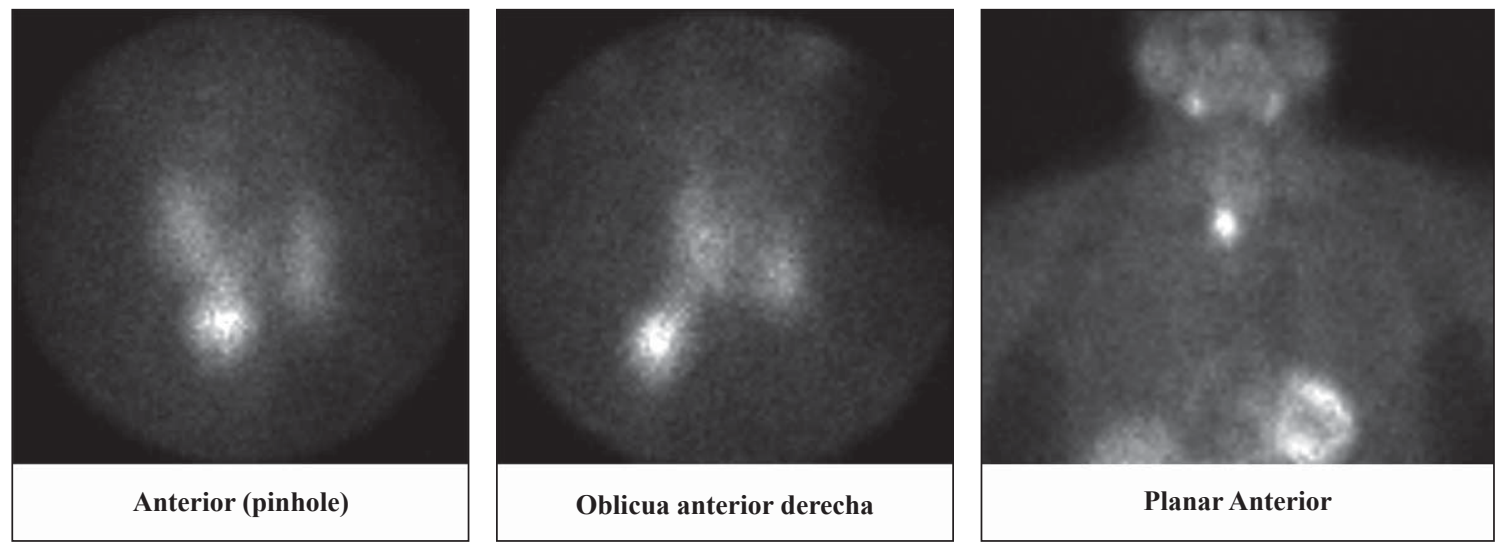

Figura 3. Caso 2: Cintigrafía de paratiroides muestra un foco hipercaptante caudal posterior derecho. 


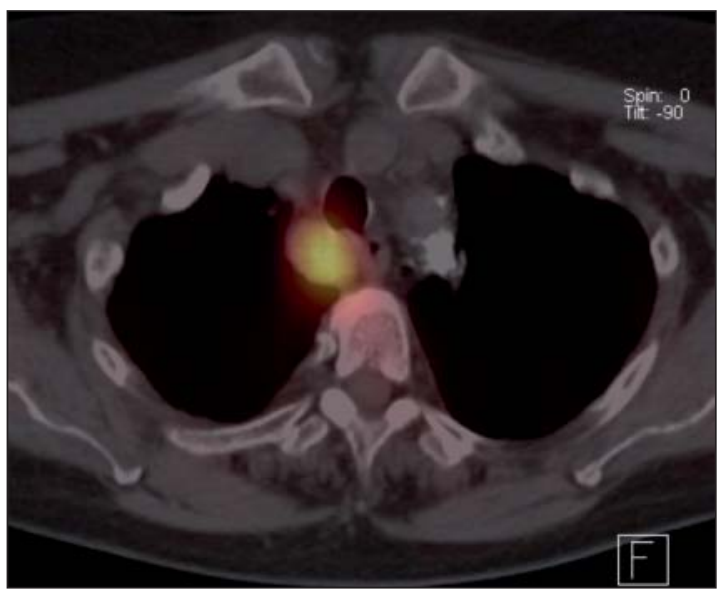

Figura 4. Caso 2: SPECT/CT con Tc99m Sestamibi precisa la localización del adenoma paratiroideo visible en la Figura 3. Adenoma paratiroideo en mediastino posterior.

conteo en el sitio de proyección del nódulo respecto al background. La PTH intacta intraoperatoria descendió de 179,9 a 27,9 pg/ml y la calcemia varió de 10,2 a $9 \mathrm{mg} / \mathrm{dl}$ en el post-operatorio. El estudio histológico confirmó una lesión encapsulada paratiroidea de 4 × 2,5 x $2 \mathrm{~cm}$ y 9,7 gramos, consistente con adenoma.

\section{Discusión}

El éxito en la localización de tejido paratiroideo hiperfuncionante requiere de un adecuado conocimiento de las posibles localizaciones, producto del desarrollo embriológico de las glándulas paratiroides. Éstas habitualmente se sitúan adyacente y posterior a los polos tiroideos, midiendo entre 1 a $5 \mathrm{~mm}$, pesando 35 gr y en un $10 \%$ pueden existir glándulas supernumerarias. Otras localizaciones menos frecuentes son, adyacente al hueso hioides, parafaríngea, escotadura traqueo esofágica, retroesofágica, adyacente a la vaina carotídea, dentro de la glándula tiroides, en el espacio posterior al manubrio esternal, en la cápsula del timo y en mediastino superior $^{8}$.

En una revisión sistemática de $20.225 \operatorname{casos}^{9}$, el $89 \%$ de los casos de hiperparatiroidismo primario correspondió a adenomas únicos, en un 4\% doble, en un $6 \%$ a hiperplasia glandular difusa y en $1 \%$ a carcinoma.

La exploración bilateral de cuello en hiperparatiroidismo primario es considerada el procedimiento estándar, con resección de todas las glándulas aumentadas de tamaño, con una tasa de éxito sobre el $90 \%$ en manos experimentadas y mínimos riesgos.
Debido a que en el hiperparatiroidismo primario predominan lesiones solitarias, la tendencia quirúrgica actual es realizar una exploración unilateral de cuello o una paratiroidectomía mínimamente invasiva $^{8}$. Ambas técnicas quirúrgicas son consecuencia de una mayor certeza diagnóstica y una adecuada localización en las imágenes pre-operatorias ${ }^{10}$, sumado a la posibilidad de efectuar PTH intraoperatoria. La paratiroidectomía mínimamente invasiva ha reducido morbilidad, costo, menos días de hospitalización y mejores resultados estéticos, especialmente cuando se realiza radioguiada con una sonda probe para detectar "in vivo" el foco hipercaptante de $99 \mathrm{mTc}$ Sestamibi ${ }^{11}$.

Entre un 5 a $10 \%$ de todos los pacientes sometidos a cirugía persisten hiperparatiroideos ${ }^{12}$, lo que puede ser consecuencia de una falla en la localización del adenoma, inadecuada resección de enfermedad multiglandular no reconocida y/o la presencia de carcinoma paratiroideo.

El ultrasonido es comúnmente realizado por su amplia disponibilidad, ser no invasivo y menor costo, pero es altamente dependiente del operador, tipo de transductor utilizado y factibilidad del paciente de extender el cuello, en ocasiones limitado en cuellos cortos, obesos y ancianos. Falsos negativos ocurren en adenomas infraesternales o retrotraqueales, en bocio multinodular o de apariencia atípica. Falsos positivos pueden ocurrir en presencia de adenopatías peritiroideas. Se describe sensibilidad en adenomas paratiroideos con ultrasonido entre 65 a $85 \%$. Sin embargo, la sensibilidad cae a $40 \%$ en pacientes con exploraciones quirúrgicas fallidas. El ultrasonido aporta además, información acerca de la coexistencia de patología tiroidea. En ambos casos, la ecotomografía convencional no visualizó la lesión paratiroidea, en el primer caso por situarse en una posición profunda del cuello y en el segundo caso por encontrarse en mediastino posterior.

En el hiperparatiroidismo persistente, en una segunda cirugía, la glándula paratiroidea anormal habitualmente se encuentra entre un 30 a $54 \%$ en el cuello, entre 16 a 34\% en mediastino, 14 a $39 \%$ retroesofágica, $5 \%$ en arco aórtico y $8 \%$ en el área cervical superior ${ }^{13,14}$. La probabilidad de éxito de una segunda cirugía sin localización es de $60 \%$ pero alcanza a un $95 \%$ cuando se tiene información de la localización de la lesión a través de imágenes ${ }^{12,15}$.

Existen actualmente varias técnicas en uso de medicina nuclear que utilizan ${ }^{99 \mathrm{~m}} \mathrm{Tc}-$ Sestamibi ${ }^{16-18}$, persistiendo controversia de cual de ellas es la óptima. Según diversos autores ${ }^{19,20}$, tanto el SPECT como las imágenes de alta resolución con pinhole son significativamente más sensibles que las imágenes planares. Un estudio comparativo entre técnicas de medicina nuclear incluyendo planares, 
SPECT, y SPECT/CT, demostró que el más alto valor diagnóstico se obtenía en los estudios en dos fases (imágenes precoces y tardías), debido a que la glándula tiroides también concentra el trazador pero tiene una depuración espontánea más rápida de modo que en las imágenes tardías de 90 a 120 minutos pueden hacerse evidentes pequeños adenomas, que no se visualizaron en las imágenes precoces, lo que se conoce por cintigrafía en 2 fases. De estos, un SPECT/CT efectuado precozmente asociado a cualquier imagen tardía era estadísticamente superior para localizar lesiones paratiroideas ${ }^{21}$.

El SPECT/CT tiene una sensibilidad similar al SPECT convencional ${ }^{22,23}$, pero es significativamente más específico ${ }^{23}$ reduciendo los falsos positivos, dada la mayor información anatómica resultado del corregistro del SPECT con el CT, agregando valor en la identificación prequirúrgica especialmente de lesiones ectópicas ${ }^{24}$.

La correcta detección de enfermedad multiglandular sigue siendo difícil. La sensibilidad para detectar enfermedad unifocal y multiglandular es de $90 \%$ y $66 \%$ respectivamente ${ }^{25}$. El peso de las lesiones es importante, bajo $600 \mathrm{mg}$ es de $86 \%$ y sobre $600 \mathrm{mg}$ es de un $94 \%$. Falsos negativos pueden ocurrir en hiperplasias o adenomas de menor volumen ${ }^{26}$.

En síntesis, la fusión de las imágenes de la tomografía computada a las imágenes de la cintigrafía con ${ }^{99 \mathrm{~m}} \mathrm{Tc}-$ Sestamibi (SPECT/CT) tiene una mayor certeza diagnóstica, siendo un valioso aporte al cirujano, permitiendo una mejor localización de lesiones paratiroideas especialmente en pacientes con cirugías previas de cuello e hiperparatiroidismo persistente o adenomas ectópicos.

\section{Referencias}

1. Sukan A, Reyhan M, Aydin M, Yapar AF, Sert Y, Campolat T, et al. Preoperative evaluation of hyperparathyroidism: the role of dual-phase parathyroid scintigraphy and ultrasound imaging. Annals of Nuclear Medicine 2008; 22:123-131.

2. Hetrakul N, Civelek AC, Stagg CA, Udelsman R. In vitro accumulation of $99 \mathrm{mTC}$ sestamibi in human parathyroid mitochondria. Surgery 2001; 130: 1011-1118.

3. Pattou F, Huglo D, Proye C. Radionuclide scanning in parathyroid disease. Br J Surg 1998; 85: 1605-1616.

4. Rubello D, Gross M D, Mariani G, Al Nahhas A. Scintigraphic techniques in primary hyperparathyroidism: from pre-operative localization to intra-operative imaging. European Journal of Nuclear Medicine \& Molecular Imaging 2007; 34: 926-933.

5. Gómez A, Gómez J, Gutiérrez MT, Expósito A, Barríos B, Zorraquino MA, y cols. Utilidad del MIBI, la ecografía y la tomografía computarizada en el diagnóstico topográfico preoperatorio del hiperparatiroidismo. Cir
Esp 2006; 80: 378-384.

6. Papathanassiou D, Flament JB, Pochart JM, Patey M, Marty H, Liehn JC, et al. SPECT/CT in localization of parathyroid adenoma or hyperplasia in patients with previous neck surgery. Clinical Nuclear Medicine 2008; 33: 394-397.

7. Harris L, Yoo J, Driedger A, Fung K, Franklin J, Gray $\mathrm{D}$, et al. Accuracy of technetium-99m SPECT-CT hybrid images in predicting the precise intraoperative anatomical location of parathyroid adenomas. Head \& Neck 2008; 30: 509-517.

8. Judson BL, Shaha AR. Nuclear Imaging and Minimamally invasive surgery in the management of hyperparathyroidism. J Nucl Med 2008; 49: 1813-1818.

9. Ruda JM, Hollenbeak CS, Stack BC Jr. A systematic review of the diagnosis and treatment of primary hyperparathyroidism from 1995 to 2003. Otolaryngol Head Neck Surg 2005; 132: 359-372.

10. Ollila DW, Caudle AS, Cance WG, Kim HJ, Cusack JC, Swasey JE, et al. Successful minimally invasive parathyroidectomy for primary hyperparathyroidism without using intraoperative parathyroid hormone assays. Am J Surg 2006; 19: 52-56.

11. Udelsman R. Six hundred fifty-six consecutive explorations for primary hyperparathyroidism. Ann Surg 2002; 235: 665-670, discussion 670-672.

12. Jaskowiak N, Norton JA, Alexander HR, Doppman JL, Shawker T, Skarulis M, et al. A prospective trial evaluating a standard approach to reoperation for missed parathyroid adenoma. Ann Surg 1996; 224: 308-320, discussion 320-321.

13. Wang CA. Parathyroid re-exploration. A clinical and pathological study of 112 cases. Ann Surg 1977; 186: 140.

14. Lange JR, Norton JA. Surgery for persistent or recurrent primary hyperparathyroidism. Curr Pract Surg 1992; 4: 26.

15. Shen W, Duren M, Morita E, Higgins C, Duh QY, Siperstein $\mathrm{AE}$, et al. Reoperation for persistent or recurrent primary hyperparathyroidism. Arch Surg 1996; 131: 861-867, discussion 867-869.

16. Sharma J, Mazzaglia P, Milas M, Berber E, Schuster D, Halkar R, et al. Radionuclide imaging for hyperparathyroidism (HPT): which is the best technetium-99m Sestamibi modality? Surgery 2006; 140: 856-863, discussion 863-865.

17. Tomas MB, Pugliese PV, Tronco GG, Love C, Palestro CJ, Nichols KJ. Pinhole versus parallel-hole collimators for parathyroid imaging: an intraindividual comparison. Journal of Nuclear Medicine Technology 2008; 36: 189194.

18. Pruzzo R, Amaral H, Morales B, Majlis S y Hurtado E. Cintigrafía de paratiroides. Rev Med Chile 1997; 125: 1182-1185.

19. Ho Shon IA, Yan W, Roach PJ, Bernard EJ, Shields M, Sywak M, et al. Comparison of pinhole and SPECT 
99mTc-MIBI imaging in primary hyperparathyroidism. Nuclear Medicine Communications 2008; 29: 949-955.

20. Nguyen BD. Parathyroid imaging with Tc-99m sestamibi planar and SPECT scintigraphy. Radiographics 1999; 19: 601-614, discussion: 615-666.

21. Lavely WC, Goetze S, Friedman KP, Leal JP, Zhang Z, Garret-Mayer E, et al. Comparison of SPECT/CT, SPECT, and planar imaging with single- and dual-phase (99m)Tc-sestamibi parathyroid scintigraphy. [erratum appears in J Nucl Med 2007;48 (9): 1430]. Journal of Nuclear Medicine 2007; 48: 1084-1089.

22. Ruf J, Seehofer D, Denecke T, Stelter L, Rayes N, Felix R, et al. Impact of image fusion and attenuation correction by SPECT-CT on the scintigraphic detection of parathyroid adenomas. Nuclear-Medizin 2007; 46: $15-21$.

23. Neumann D, Obuchowski N, DIFilippo F. Preoperative
I123/99mTc-Sestamibi Sustraction SPECT and SPECT/ CT in primary Hiperparathiroidism. J Nucl Med 2008; 49: 2012-2017.

24. Gayed I W, Kin E, Broussard W, Evans D, Lee J, Broemeling LD, et al. The value of $99 \mathrm{~m}$ Tc-Sestamibi SPECT/CT over Conventinal SPECT in the evaluation of parathyroid adenomas or hiperplasia. J Nucl Med 2005; 46: 248-252.

25. Nichols K, Tomas M, Tronco G, Rini J, Kunjummen B, Heller K, et al. Preoperative parathyroid scintigraphic lesion localization: accuracy of various types of readings. Radiology 2008; 248: 221-232.

26. Chiu B, Sturgeon C, Angelos P. What is the link between nonlocalizing sestamibi scans, multigland disease, and persistent hypercalcemia? A study of 401 consecutive patients undergoing parathyroidectomy. Surgery 2006; 140: 418-422. 